Paper

\title{
Study on Optimal Lighting Configuration and Aberration of Inspection System by Monte Carlo Method
}

\author{
L. CHEN, Masafumi SUZUKI and Noboru YOSHIMURA
}

Dept. of E. \& E. Eng, Akita University

Tegatagakuen 1-1, Akita 010-8502, Japan

Received May 27, 1998.

\begin{abstract}
The improvement of image quality has a close relation with lighting and lens in an automatic inspection system. The quality of image could affect the cost, speed and stability of an inspection system. The influences of the lighting configuration and the spherical aberration of lens in an automatic inspection system are studied by means of Monte Carlo (MC) method in this paper. The theoretical analysis and simulation results reveal as follows: when the light source deviates from the center of the inspection system, the planar light source with a large (small) inclination has a better illumination in the near (far) distance within a narrow (broad) scope. The illumination scope becomes broader as the sloped light source deviates from the center of the inspection system. There exist some symmetric distortions in the margin of the pattern while the spherical aberration presents. This research not only supplies an available method for the analysis of lighting configuration but also points out a new way for analyzing the aberration of lens.
\end{abstract}

\section{Introduction}

Light source and inspection lens play an important part in detecting the internal drawback and outward appearance of the inspected object. The lighting technique and the detecting device determine the quality, cost, speed and stability of the inspection system in an automatic inspection system. With the development of automatic inspection systems, it becomes more and more important that the influences of the lighting configuration and the aberration of lens on the inspection results are examined. At present, the most methods in service are analytics and measuring methods, and some researches on the analysis of the illumination effect of light source have being presented using these methods ${ }^{1-4)}$. However, it is complicated that the relation between the inspected object and the light source is reflected either by analytics or by measuring methods. This is because no matter where the light source is located and how the inspected object is detected, the inspection results are simultaneously affected by such as the lighting configuration, the reflectance of inspected object, and the performance of lens, so that the analytic results of an inspection systems cannot be easily determined by finding the resolutions of differential or integral equations of light transport. Generally speaking, the analytics are often too tedious to use, and the measuring methods cannot express the effect of illumination in an automatic inspection system completely ${ }^{4-6)}$. On the other hand, the aberration of lens varies from one lens design to another; two different lenses with identical properties at their design conjugates may behave quite differently if used at the wrong conjugates ${ }^{7)}$. Therefore, it is difficult to analyze the influence of lens aberration numerically.

MC method is a well known technique in application where finding the direct resolution is very difficult. It is used as a research tool stems from work on the atomic bomb during the second world war. The possibility of applying Monte Carlo methods to deterministic problems was noticed by Fermi, von Neumann, and Ulam and popularized by them in the immediate post-war years. About 1948 Fermi, Metropolis, and Ulam obtained Monte Carlo estimates for eigenvalue of the Schrodinger equation ${ }^{8)}$. In the last decade, lighting researches have investigated the potential of the Monte Carlo technique for lighting calculations in an effort to overcome some drawbacks of finite element methods. Tregenza $^{9)}$ and Stanger ${ }^{10)}$ developed techniques for the application of the method in lighting. As it has been demonstrated by lighting researches, such as Kinameri ${ }^{11)}$, Kajiyama, Kodaira ${ }^{12)}$, Aoki, Taniguchi, Irikura ${ }^{13)}$, Uchida and Ohtani ${ }^{14)}$, good agreement between computed and measured results was claimed. In general, the simulation methods are analysis tools for specialist applications and research and as such were neither suitable for, or available to, practising designers ${ }^{15)}$. Our model is just the one which needs to be investigated by simulation method. This is because the model dimension is so small that there are some 
difficulties in selecting measuring points and in ensuring measuring accuracy; moreover, since the aberration of lens varies from one lens design to another, measuring results might not be of universal significance. The simulation method is reasonable for this research.

Generally speaking, in lighting research, the reflection performance of the illuminated object is the one of the most important factors. The light reflected at the interface between air and another material has two componentslight reflected by the surface and light reflected by the bulk of the material ${ }^{16-18)}$, that is, no matter what material and what shape of the illuminated object are, the method which treats light reflected at the interface is similar. Without loss of generality, we take an IC substrate as the inspected model to investigate the reflection mechanism of luminous flux, the relationship between the lighting configuration and the inspection object, and the influence of spherical aberration of lens.

\section{Simulation Principle and Model}

\subsection{Principle}

The cardinal principle of Monte Carlo method is the one which uses random sampling to solve a problem which can be modeled by probability method ${ }^{9)}{ }^{10)}$. MC method provides an answer to a problem in terms of the number of photon bundles which have been reflected from each element of surface area divided by the number of total walks started. These numbers are the ratio of light on the surface to total light emitted by the source. In this method, each set of random emission is an experiment. After the scaled emission times are finished, the luminous exitance $M$ of each element is defined by the following equation ${ }^{19)}$ :

$$
M^{\prime}=\frac{A}{P} \times \frac{F}{S_{A}}
$$

Where, $M^{\prime}$ is the luminous exitance of each element, $A$ is the number of the photon bundles which the element reflects, $F$ is the total luminous flux, $S_{\wedge}$ is the area of an element, and $P$ is the total number of photon bundles which the light source emits.

As a scale of Eq. 1, the total luminous flux does not affect the feature of the inspecting results, thus we can normalize $M$ by $F$,

$$
M=\frac{A}{P} \times \frac{1}{S_{A}}
$$

Eq. 2 shows that the number of reflected photon bundles can exhibit the luminous exitance of each element. Therefore, it is used to evaluate the performance of inspection system in this paper.

\subsection{Model}

The inspection system is illustrated in Fig.1. The lighting is composed of two inclined planar light sources and the inspected object is an IC substrate. It is assumed that if the incident ray is reflected by the substrate and received by the lens, it will reach $\mathrm{CCD}$ detector. The simulation mode is shown in Fig.2.

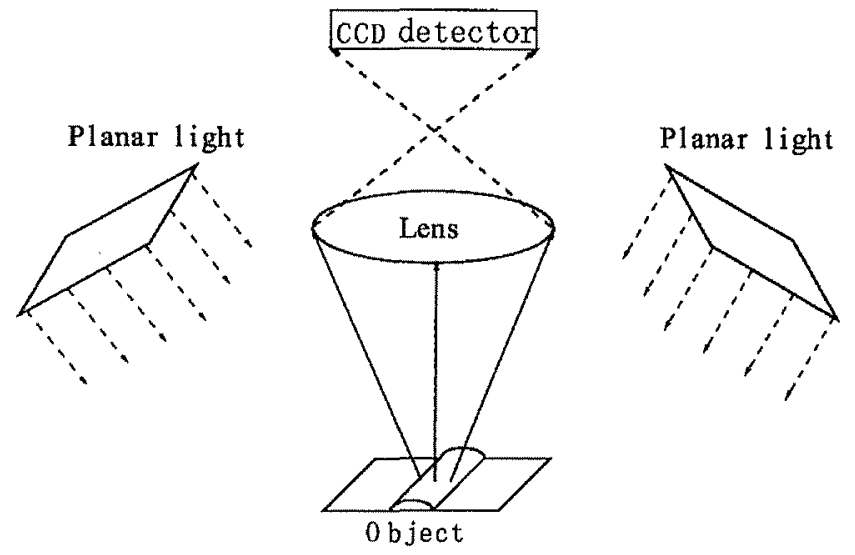

Fig.1 The model of an inspection system.

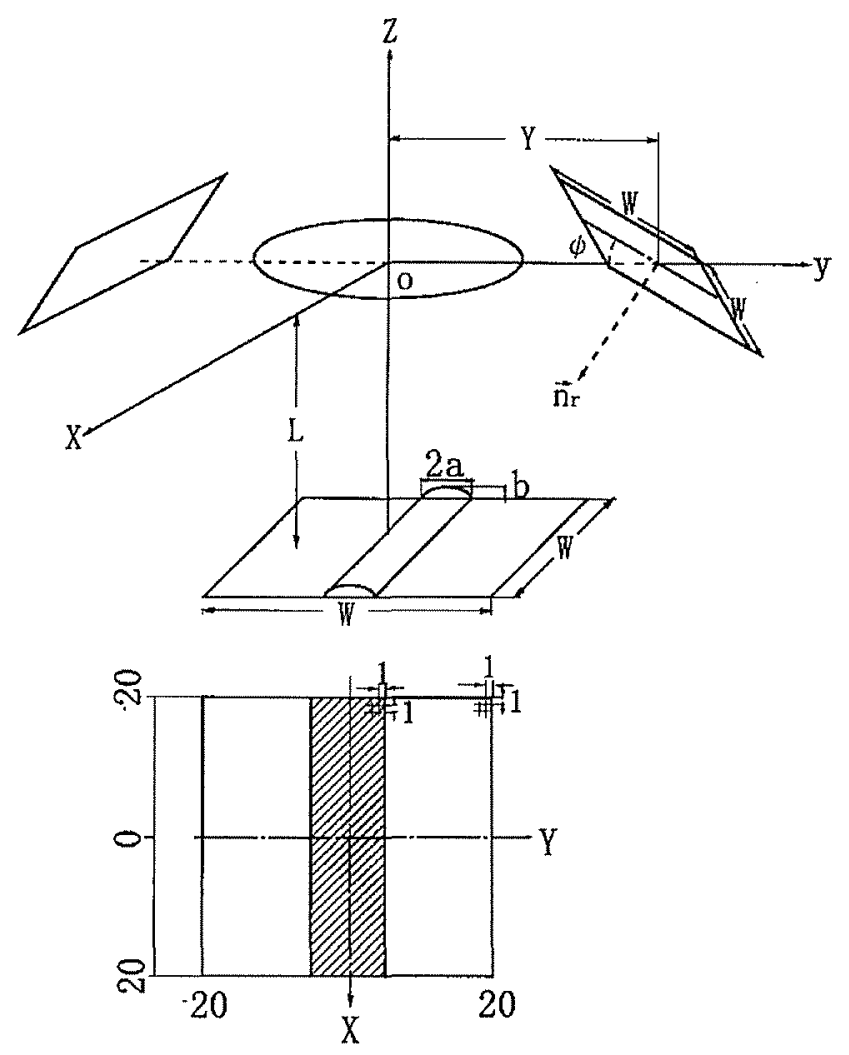

Unit mm

Fig.2 The simulation model. $\psi$ is the slope of the light source, $W$ is the side of the light source. "a" and "b" refer to half the length of minor and major axes of the printed wire, and $a=5, b=1 \mathrm{~mm}$.

The light sources and the inspected object are defined as a square whose side is $W$. The distance from the lens to the inspected object and the distance which the light source 
deviates from the center of the inspection system are $L$ and $Y$, respectively. $L, Y$ and $W$ are taken as $40 \mathrm{~mm}$ in this study. The printed wire on IC substrate is assumed to be a half elliptic cylinder which is located on the middle of the substrate. The width and thickness of the printed wire are assumed to be $2 a$ and $b$, respectively. The printed wire is divided into 400 elements. The residual parts of the substrate, which are located on the both sides of the printed wire, are divided into 600 elements respectively. Every element of the board and the wire is a square millimeter in the $x y$ plane (see the bottom of Fig.2).

\section{Simulation Procedure}

The simulation mainly includes the following procedures: Firstly, the emission direction and position of a photon bundle emitted from the light source are determined. Secondly, when a photon bundle strikes the surface, absorption or reflection is decided by random number and the reflection direction is determined here. Lastly, if the reflected photon bundle is collected by the lens, the spherical aberration of lens is determined with a random number and the element which corresponds to the image receiver is incremented or deducted according to the type of spherical aberration.

\subsection{Emission of Light Source}

The emission point of a photon bundle from the light source (see Fig.2 and appendix [1]) is:

$$
\left\{\begin{array}{l}
x_{0}=\xi W-\frac{W}{2.0} \\
y_{0}=\zeta W \cos \psi-\frac{W \cos \psi}{2.0} \pm Y \\
z_{0}=\kappa W \sin \psi-\frac{W \sin \psi}{2.0}
\end{array}\right.
$$

The emission equation of a photon bundle is:

$$
\left\{\begin{array}{l}
x=x_{0} \\
z=z_{0}+\left(y-y_{0}\right) \operatorname{ctg} \psi
\end{array}\right.
$$

\subsection{Incident Point of Photon Bundle}

The incident point of a photon bundle on the board:

$$
\left\{\begin{array}{l}
\mathrm{x}=\mathrm{x}_{0} \\
y=y_{0} \pm \frac{L+z_{0}}{\operatorname{tg} \psi} \\
z=-L
\end{array}\right.
$$

Where, the sign of $\operatorname{tg} \psi$ selected according to the location of the light source.

According to the equations of a photon bundle and the printed wire, the incident point of a photon bundle on the printed wire is determined as follows

$$
\left\{\begin{array}{l}
x=x_{0} \\
y=\frac{-B \pm \sqrt{B^{2}-4 A C}}{2 A} \\
z=\frac{a}{b} \sqrt{b^{2}-y^{2}}-L
\end{array}\right.
$$

Where, $a$ and $b$ are half the length of minor and major axes of the printed wire respectively. The sign of the square root depends on the location of the light source.

\subsection{Absorption or Reflection of Incident Light}

When a photon bundle strikes the surface, the reflection or absorption should be decided. Since all the reflectances and absorptances of the surface are known numbers, the diffuse reflection, specular reflection, or absorption can be determined by a random number $\rho$ (see appendix [2)).

$$
\begin{array}{ll}
\text { diffuse reflection } & 0<\rho \leq \rho_{\mathrm{d}} \\
\text { specular reflection } & \rho_{\mathrm{d}}<\rho \leq \rho_{\mathrm{d}}+\rho_{\mathrm{s}} \\
\text { absorption } & \rho_{\mathrm{d}}+\rho_{\mathrm{s}}<\rho<1
\end{array}
$$

Where, $\rho_{\mathrm{d}}$ is the diffuse reflectance, and $\rho_{\mathrm{s}}$ is the specular reflectance.

In specular reflection, light is reflected in an equal and opposite direction to the angle of incidence. Diffuse reflection occurs when the reflection is independent of the angle of incidence. In the case of diffuse reflection, according to Lambert's cosine law, the luminous intensity $I_{\theta}$ in $\theta$ direction is

$$
I_{\theta}=I_{n} \cos \theta
$$

Where, $I_{n}$ is the luminous intensity in the direction of surface normal.

The probability of diffuse reflection in $\theta$ direction can be expressed as follows:

$$
F(\theta)=F(0) \cdot \cos \theta
$$

Where, $F(0)$ is the reflection probability in the regular direction. Solving for cone angle $\theta$ yield

$$
\begin{aligned}
\eta & =\int_{0}^{\theta} F(0) \cos \theta \sin \theta \mathrm{d} \theta=\int_{0}^{\theta} \frac{1}{2} \sin 2 \theta \mathrm{d} 2 \theta \\
\theta & =\frac{\cos ^{-1}(1-2 \eta)}{2}
\end{aligned}
$$

Where, $\eta$ is a random number.

Since the reflection in the circumferential angle direction is equally probable, the circumferential angle $\phi$ can be defined as

$$
\phi=2 \pi \xi
$$

In the case of specular reflection, the intensity and the direction of reflection depend on incidence angle and 
surface roughness. According to the Law of Reflection, the reflection vector $\boldsymbol{R}\left(\cos \alpha_{\mathrm{r}}, \cos \beta_{\mathrm{r}}, \cos \gamma_{\mathrm{r}}\right)$ can be decided in the same way as ${ }^{201}$. Based on the measured results of the spectrometer JUKI JP7100P, we take the diffuse and specular reflectance and the absorptance of the board are $0.6,0.3$ and 0.1 , and the diffuse and specular reflectance and the absorptance of the wire are $0.2,0.7$, and 0.1 in this simulation.

\subsection{Aberration of the Lens}

Aberration of lens has many manifestations, such as "wavefront aberration," "ray aberration," etc. However, these terms do not refer to fundamentally different things, but to different aspects of the same thing. Often, a single manifestation of the aberration is considered, according to what is measurable, what best describes the degradation in a particular application. It is well known that the spherical aberration is a function of its shape factor or bending and that when the $F$-number, refractive index and object-image distance are fixed, the spherical aberration is caused by the difference in distance of which the incident ray is away from the optic axis ${ }^{21}$. That is, under this situation, when the ray emanates from a point of the optic axis, the intersection of outgoing ray on the optic axis varies according to the position of incident point. The difference of the intersection point results in what is called spherical aberration. Usually, the spherical aberration is determined by the displacement $\delta$, as shown in Fig. 3 .

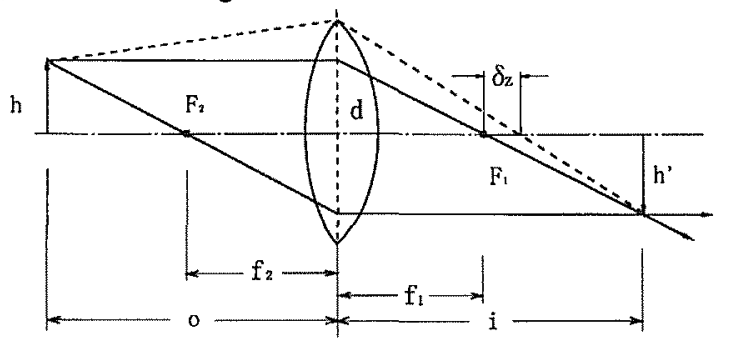

Fig.3 The image location and spherical aberration. $d$ is the refraction index of lens, $\delta_{z}$ is the displacement caused by spherical aberration, 0 and $i$ are the object and image distances, focal length $f_{1}$ and $f_{2}$ are taken the same and denoted by $f$.

It has been shown experimentally that, for the majority of objectives, the amount of spherical aberration in focused spot is directly proportional to the displacement $\delta_{z}{ }^{7}$. In the manner of Hopkins ${ }^{22}$, the $\delta$, is related to parameter $W_{20}$ which is used to measure aberration of lens, as shown in Fig.4.

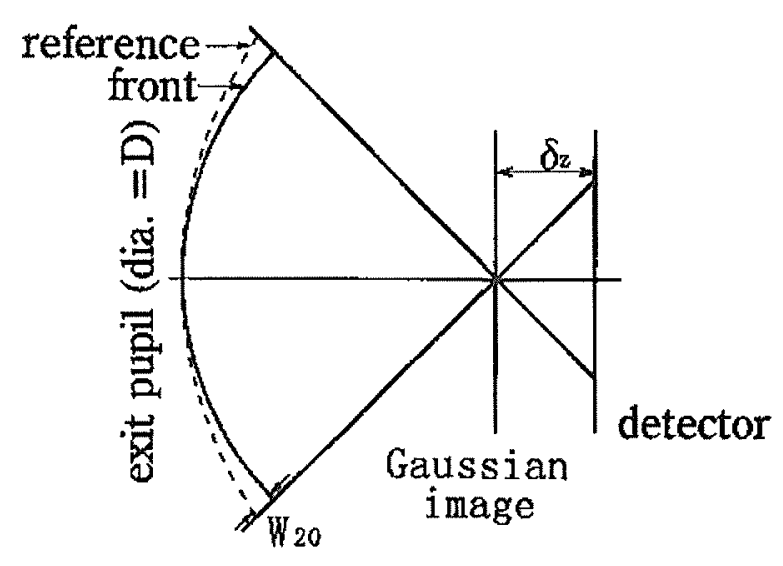

Fig.4 Measuring spherical aberration

$W_{20}$ equals the greatest optical path-length error between the wave front and a reference sphere whose origin is the true image point lying in the detector plane. The path length $W_{20}$ is related to the distance $\delta_{z}$, the diameter of the exit pupil $D$, and the image distance $i$ by ${ }^{23-25)}$.

$$
\mathrm{W}_{20}=\frac{1}{2}\left(\frac{\mathrm{D}}{2 i}\right)^{2} \delta_{z}
$$

We assume that the object is illuminated with spatially incoherent, quasi-monochromatic light and that only spherical aberration exists. The pupil function related to the spherical aberration of lens is decided by ${ }^{23}$

$$
\mathrm{P}_{\mathrm{R}}(\nu)= \begin{cases}1 & \varepsilon \leqq \nu \leqq 1 \\ 0 & \text { otherwise }\end{cases}
$$

Where, $\varepsilon \in[0,1)(\varepsilon \propto \delta$, ) is the normalized radius of the obscured region of the pupil as a fraction of the pupil's outer radius, $v$ is a random number.

When a photon bundle falls on the lens, according to the value of $P_{\mathrm{R}}(v)$, we decide whether the spherical aberration takes place or not. If yes, the element which corresponds to the image receiver is processed by random number. Then, the element which corresponds to the image receiver is incremented or deducted according to the type of spherical aberration.

\subsection{Location of the Image and Collection of Photon Bundles by the Lens}

Since the reciprocal of the focal length of a thin lens combination is equal to the sum of the individual lenses, the reciprocal can be simplified to be a single thin lens. The image location can be found from Fig.3.

$$
\left|h^{\prime}\right|=\frac{|f||h|}{|a-f|}
$$


Where, $h$ and $h^{\prime}$ are the distances that the start point of the reflected photon bundle and its image location are away from the $z$ axis respectively.

\section{Results and Discussion}

In this research, we examine the effect of the lighting configuration under the situation that the inspection system is in focus $\left(\delta_{2}=0\right)$. Likewise, the spherical aberration of lens is investigated under a sloped light source (slope $=25^{\circ}$ ). In this research, it is assumed that the $F$-number is 0.5 , the refractive index of lens is 1.5 and object-image distance is equal, and the spherical aberration is caused by the difference which the incident point is away from the optic axis.

\subsection{Influence of the slope of the light source}

When the distance between the lens and the inspected object keeps constant (say, $40 \mathrm{~mm}$ ), the relation between the collected photon bundles (assembly average) and the slope of the light source is illustrated in Fig.5.

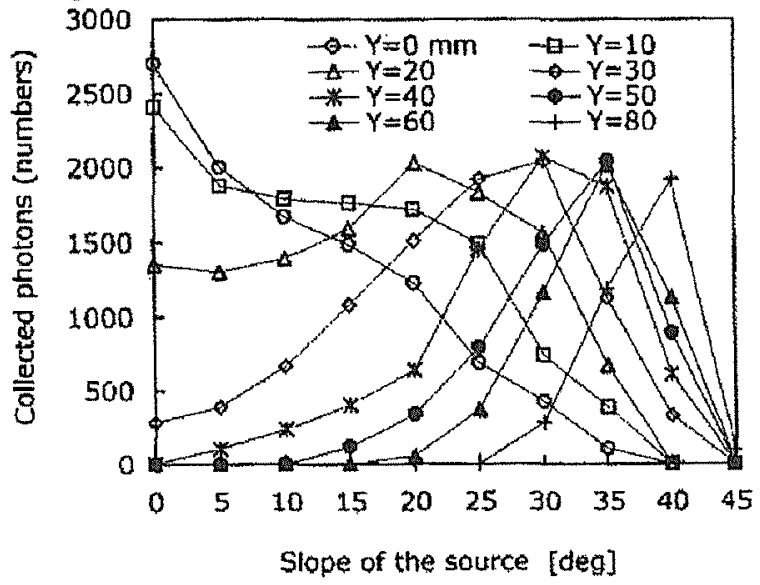

Fig.5 The relation between the number of collected photon bundles and the slope of the light source. $y=0$ refers to that the light source is directly over the inspection center. $y=10$ means that the light source deviates the inspection center $10 \mathrm{~mm}$ and so forth.

It is shown that when the slope of the light source is small, the light source with no (or little) deviation can results in a better illumination. Along with the increase of the slope, the illumination caused by such a light source drops dramatically. Otherwise, with the slope increasing, a farther deviated light source (i.e., $Y$ is large) could create a better illumination. It is known that no matter what the slope of the light source is, the maximum illumination changes little, i.e., the optimal illumination can be gotten at any slope of the light source. It is noted that, if the slope is the same, the farther offseted light source can cause the illumination changing much sooner. For example, when $Y=30 \mathrm{~mm}$, slope $=35^{\circ}$, we increase the slope $5^{\circ}$, the collected photon bundles decrease by less than a half of the maximum illumination. Accordingly, when $Y=50 \mathrm{~mm}$, slope $=35^{\circ}$ (the light source is farther from the inspection center than the former), the collected photon bundles decreased by more than a half while we increase the slope $5^{\circ}$. That is, the farther the light source away from the center of the ordinate system, the sooner the photon bundles decrease from the largest to the lowest value. In practice, if the light source is far way from the inspection center, one should pay more attention to adjusting the slope of the light source, because in this situation, the effectual illumination range is narrow, a slightly adjustment of the slope will affect the illumination significantly.

\subsection{Optimal lighting distance}

In this research, we regard the region in which the illumination drops from the largest value to a half value as the illumination range ${ }^{26}$. When we hold the distance which the light source offsets the inspection center fixed as $40 \mathrm{~mm}$ (i.e., $Y=40$ ) and vary the slope of the light source, the relation between the distance and the collected photons is shown in Fig.6.

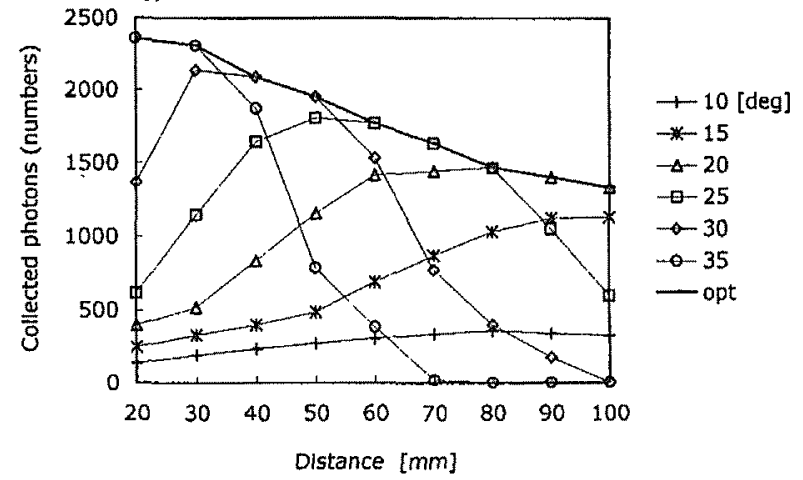

Fig.6 The relation between the collected photon bundles and the inspection distance when the light source deviates from the inspection center $40 \mathrm{~mm}$.

Let us observe the illumination situation at two case: one is the slope being $35^{\circ}$, the other is the slope being $25^{\circ}$. It is know that in the former case, the illumination range is about $25 \mathrm{~mm}$, i.e., if we vary the inspection distance of the inspected object within this range, a good illumination can be gotten. Accordingly, in the later case, the illumination range is about $60 \mathrm{~mm}$-broader than the former. When the inspection distance is $20 \mathrm{~mm}$, we get the optimal illumination with the slope $35^{\circ}$. Similarly, when the inspection distance is $50 \mathrm{~mm}$, we get the optimal illumination with the slope $25^{\circ}$. Moreover, it is noted that when the optimal illumination is gotten in the former case (photon bundles are about 2360), the photon bundles in the later is only about 610 -almost a quarter of the optimal illumination. Likewise, when the optimal illumination is gotten in the later (photon bundles are about 1810), the photon bundles in the former is only about 790-almost a third of the optimal illumination. It is clear that in the far distance, a small slope makes the illumination better and broader than that of the large slope, but as the light source 
deviates from the inspection center, the illumination by a small sloped source will be very poor. For example, when the slope of the light source is $10^{\circ}$, we cannot get good illumination almost in all distances (the photon bundles reflected to the lens are less than 400 -much fewer than the maximum illumination). The figure exhibits that the range of optimal illumination varies with the slope and that under a larger slope, the illumination distance can scarcely be adjusted. That is, the larger slope results in a narrower illumination range. It is known that a given slope angle can remain the optimal illumination in some distance, but it cannot keep the optimal illumination in all distances. Thus, if we want to get the optimal illumination in the far distance, we have to restrain the slope being small; otherwise, we need to make the slope of the light source larger to get the optimal illumination in the near distance.

In order to get the optimal illumination in all distances, we connect the maximum points of every curve, shown as in Fig.6. The optimal lighting in different distances can be gotten as the slope varies. It is noted that, under the optimal illumination, the illuminance obeys an inverse square law. In practice, in order to get the optimal illumination in different distances, the slope of the light source should vary to meet the need of the inspection.

\subsection{Influence of the light source departure}

If we hold the distance between the lens and the inspected object fixed ( $40 \mathrm{~mm}$, for instance), and let the light source be away from the inspection center, the relation between the collected photon bundles and the light source deviation is shown in Fig.7.

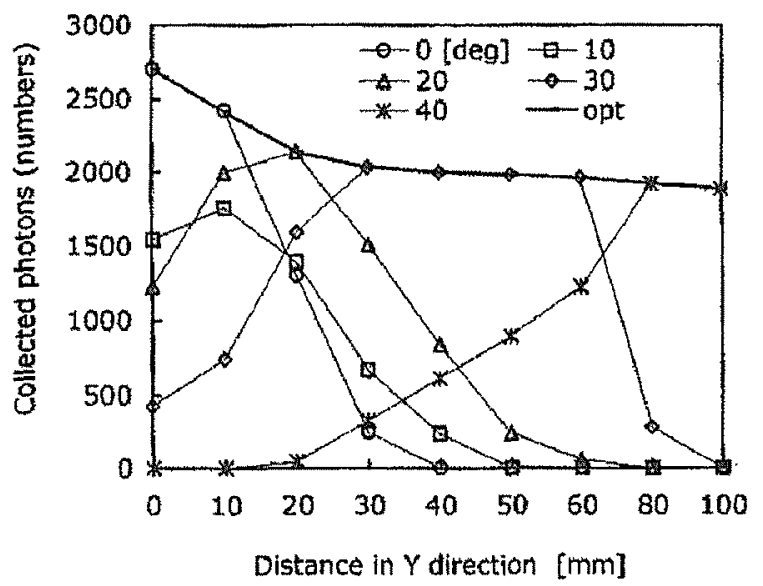

Fig.7 The collected photon bundles when the light source deviates from the inspection center with the different sloping angles.

It is seen that the illumination of the small (or zero) sloped light source drops dramatically as the light source deviates from the inspection center. On the other hand, if the slope of the light source increases, the illumination can remain constant in a relatively large range as the light source is far away from the inspection center. This is because the sloped light source has a larger illumination region (seen in Fig.2).
Since the sloped light source can result in a larger illumination region on the inspected object, so that the illumination from a sloped light source decreases little in a relatively broad range as the light source deviates from the inspection center. This result implies that when the light source deviates from the inspection center, the slope angle of the light source should be given to keep the optimal illumination in a relatively larger range. If we connect the maximum points of every curve, the optimal lighting slope for the different departure can be gotten, as shown in Fig.7. Comparing it with Fig.6, it is evident that although they both obey an inverse square law, the decreasing tendency in Fig.7 is slower than Fig.6. The reason is that the drop of collected photon bundles caused by the distance variation is much larger than that caused by the deviation from the inspection center (see Fig.2).

\subsection{Influence of the aberration}

When $\delta_{z}$ is equal to zero, that is, the lens has no spherical aberration, under a sloped light source (sloped angle is $25^{\circ}$ ), the collected photon bundles are shown in Fig.8.

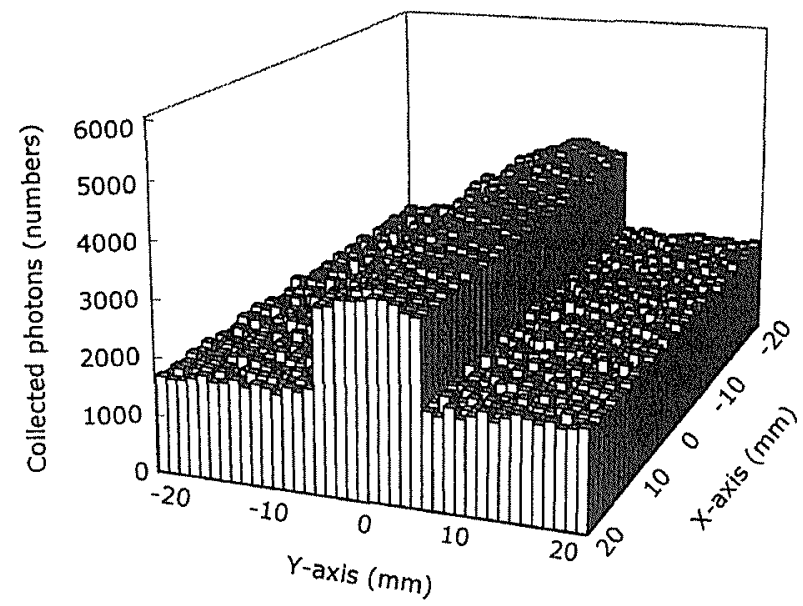

Fig.8 The collected photon bundles without the aberration $\left(\delta_{z}\right.$ is 0$)$.

It is shown that the collected photon of lens is evenly distributed. Namely, in this situation, the inspected pattern can be transferred with no distortion. When $\delta$, is negatively biased and is equal to one tenth of the curvature radius of the lens, there exist some symmetric swell distortion in the margin of the pattern, as shown in Fig.9.

Fig. 10 shows the situation when $\delta$, is positively biased. It exhibits there exist some symmetric sag distortion in the margin of the pattern. Comparing it with Fig.9, the distortions are reversed. We thus can conclude the corollary: the modulation of spherical aberration proportions $\delta_{2}$ and it is possible the spherical aberration of the reciprocal is cancelled. Namely, the two lenses see equal but opposite displacements, $\delta$, and $-\delta_{x}$, these spherical aberrations can be exactly cancelled each other. 


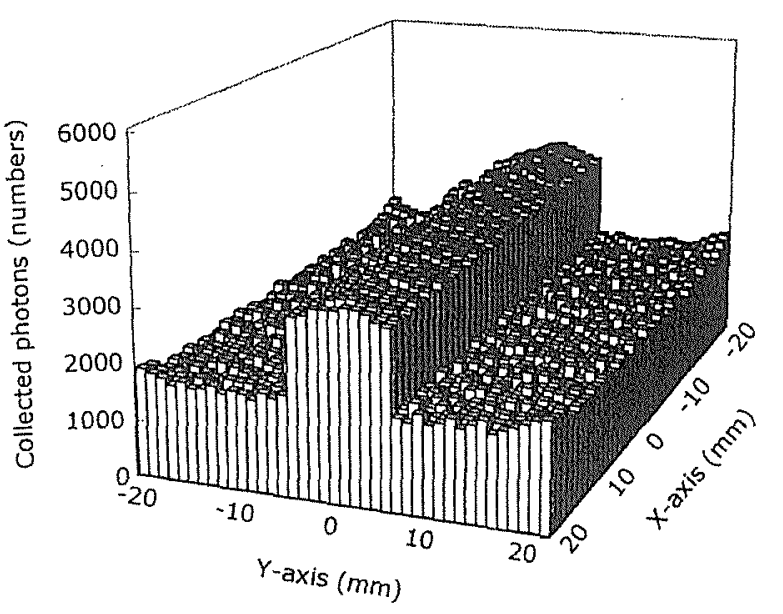

Fig.9 The collected photon bundles when $\delta_{z}$ is equal to one tenth of the curvature radius of lens and negatively biased.

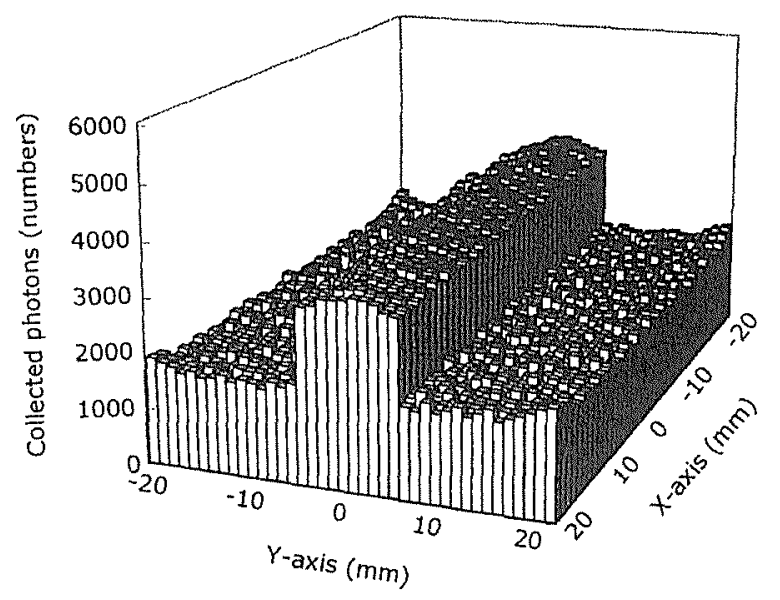

Fig.10 The collected photon bundles when $\delta_{z}$ is the same as Fig9, but positively biased.

This corollary has directive function in the production and the use of the inspection devices-one can make a good image by cancelling the spherical aberration of the reciprocal and the inspection result can become better by using the lenses that see equal but opposite displacements.

\section{Conclusions}

The study in this paper shows that it is possible that the lighting configuration and the performance of optics system are studied by MC method. A greater (smaller) slope of the light source has a better illumination in the near (far) distance and that the greater the slope of the light source, the narrower the illumination scope. When the light source deviates from the center of the inspection system, a sloped light source can result in a larger illumination region for the inspected object. When the spherical aberration presents, some symmetric distortions appear in the margin of the pattern, and it is directly proportional to the displacement on the optical axis.

\section{Appendix}

(1) Deviation of Eq. 3 to Eq. 6

If we express the emerging point of the planar light source $(\mathrm{x} 0, \mathrm{y} 0, \mathrm{z} 0)$ with random numbers, we obtain,

$$
\left\{\begin{array}{l}
x_{0}=\xi W-\frac{W}{2.0} \\
y_{0}=\xi W \cos \psi-\frac{W \cos \psi}{2.0} \pm Y \\
z_{0}=\kappa W \sin \psi-\frac{W \sin \psi}{2.0}
\end{array}\right.
$$

where, $Y$ is the distance of the light source center from the origin of the coordinate system. When the light source is located at the positive direction of the ordinate, $Y$ takes the positive sign; otherwise, it takes the negative sign. $\xi, \xi$ and $\kappa$ are random numbers whose values are from 0 to 1 . As shown in Fig.2, $x_{0}$ is determined by $\xi$. According to $\xi$ being 0 or $1, x_{0}$ takes its minimal value $-W / 2$ or maximal value $W / 2$. When $\xi$ is between 0 to $1, x_{0}$ is between $-W / 2$ to $W / 2$. In the same way, we can determine the values of $y_{0}$ and $z_{0}$. Since the source light plane normal is not vertical to the $y$ and $z$ axes, the slope angle of the source light plane $\psi$ is included in the expressions of $y_{0}$ and $z_{0}$.

From Fig.2, it is known that the direction vector of a photon emerging from the light source is,

$$
\begin{aligned}
& \overrightarrow{n_{r}}=0 \cdot \vec{i}-\cos \psi \vec{j}-\sin \psi \vec{k} \\
& \vec{n}_{r}=\left(x-x_{o}\right) \vec{i}+\left(y-y_{o}\right) \vec{j}+\left(z-z_{o}\right) \vec{k}
\end{aligned}
$$

Also, $\overrightarrow{n_{r}}$ can be expressed as

$$
\begin{aligned}
& \overrightarrow{n_{r}^{0}}=\left\{\frac{x-x_{0}}{\Delta}, \frac{y-y_{0}}{\Delta}, \frac{z-z_{0}}{\Delta}\right\} \\
& \Delta=\sqrt{\left(x-x_{0}\right)^{2}+\left(y-y_{0}\right)^{2}+\left(z-z_{0}\right)^{2}}
\end{aligned}
$$

That is

$$
\left\{\begin{array}{l}
\frac{x-x_{0}}{\Delta}=0 \\
\frac{y-y_{0}}{\Delta}=-\cos \psi \\
\frac{z-z_{0}}{\Delta}=-\sin \psi
\end{array}\right.
$$

$(\mathrm{g}):(\mathrm{f})$, we obtain the equation of a emerging photon as follows

$$
\left\{\begin{array}{l}
x=x_{0} \\
z=z_{0}+\left(y-y_{0}\right) \operatorname{tg} \psi
\end{array}\right.
$$


The intersection of a photon bundle with the board can be extracted from the simulaneous equation of the both, that is,

$$
\left\{\begin{array}{l}
\mathrm{x}=\mathrm{x}_{0} \\
\mathrm{z}=\mathrm{z} 0+\left(\mathrm{y}-\mathrm{y}_{0}\right) \operatorname{tg} \psi \\
\mathrm{z}=-L
\end{array}\right.
$$

The intersection on the board is

$$
\left\{\begin{array}{l}
\mathrm{x}=\mathrm{x}_{0} \\
y=y_{0}-\frac{L+z_{0}}{\operatorname{tg} \psi} \\
\mathrm{z}=-L
\end{array}\right.
$$

In the same way we can find the intersection of a photon bundle with the wire as the following,

$$
\left\{\begin{array}{l}
\mathrm{x}=\mathrm{x}_{0} \\
\mathrm{z}=\mathrm{z}_{0}+\left(\mathrm{y}-\mathrm{y}_{0}\right) \operatorname{tg} \psi \\
\frac{y^{2}}{b^{2}}+\frac{(z+L)^{2}}{a^{2}}=1
\end{array}\right.
$$

That is,

$$
\left\{\begin{array}{l}
x=x_{0} \\
z=z_{0}+\left(y-y_{0}\right) \operatorname{tg} \psi \\
a^{2} y^{2}+b^{2}(z+L)^{2}=a^{2} b^{2}
\end{array}\right.
$$

Let $c_{1}=z_{0}+L-y_{0} \operatorname{tg} \psi$ and substituting (k) into (l), we arrive at, $\left(a^{2}+b^{2} \operatorname{tg}^{2} \psi\right) y^{2}+2 c 1 \operatorname{tg} \psi b^{2} y+b^{2} c 1^{2}-a^{2} b^{2}=0$

$$
\text { Let, } \quad \begin{aligned}
A & =\mathrm{a}^{2}+\mathrm{b}^{2} \operatorname{tg}^{2} \psi \\
B & =2 \mathrm{c}_{1} \mathrm{~b}^{2} \operatorname{tg} \psi \\
C & =\mathrm{b}^{2} \mathrm{c}^{2}{ }^{2}-\mathrm{a}^{2} \mathrm{~b}^{2}
\end{aligned}
$$

We obtain the intersection on the wire,

$$
\left\{\begin{array}{l}
\mathrm{x}=\mathrm{xo} \\
y=\frac{-B \pm \sqrt{B^{2}-4 A C}}{2 A} \\
z=\frac{a}{b} \sqrt{b^{2}-y^{2}}-L
\end{array}\right.
$$

Where, the sign in front of the square root is decided by the location of the light source. If it locates at the positive direction of $y$ axis, the positive sign is taken.

(2) About Eq.7 to 12 , please see the references $(19,26$, 27).

\section{References}

(1) Kajiya J. T. : "The Rendering Equation", Computer Graphics (SIGGRAPH'86 Proc.), Vol. 20, pp. 143150(Aug. 1986).
(2) Ward, G. J. and Rubenstein, F. : "A Ray Tracing Solution for Diffuse Interreflection", Computer Graphics (SIGGRAPH'88 Proc.), Vol. 22, pp. 85-92 (Aug. 1988.)

(3) Sillion, F. X., Arvo, J. R., Westin, S. H. and Greenberg, D. P. : "A Global Illumination Solution for General Reflectance Distributions", Computer Graphics (SIGGRAPH'91 Proc.), Vol. 25, pp. 187-196 (July. 1991).

(4) Kinameri, K., Kashioka, S., Nonaka, M. and Miyatake, T. : "Evaluation on the Illuminance of the Image of the Imperfectly Diffused Reflective Surface by Using a Microscope", Proc. of 1980 Annual Conf. of Illum. Engng. Inst. Jpn., pp. 115-116 (1980).

(5) Max, N. : "Optical Models for Direct Volume Rendering", IEEE TVCG, Vol. 1, No. 2, pp. 99-107 (1995).

(6) Zagajac, J. : "A Fast Method for Estimating Discrete Field Values in Early Engineering Design", IEEE TVCG, Vol. 2, No. 1, pp. 35-43 (1996).

(7) Gerber, E., Mansuripur, M. and Sasian, J. M. : "Versatile Objective Lens with Adjustable Correction for Different Wavelengths and Substrate Thicknesses for Testing Optical Disks", OSA. Applied Optics, Vol. 36, No. 11/10, pp. 2414-2420 (1997).

(8) Hammersley J. M., and Handscomb, D. C. : "Monte Carlo Methods", London: Methuen \& Co Ltd, Chapter one (1967).

(9) Tregenza, P. R. : "The Monte Carlo Method in Lighting Calculations", Lighting Research \& Technology, Vol. 15, No. 4, pp. 165-170 (1983).

(10) Stanger, D. : "Monte Carlo procedure in lighting design", Journal of the Illuminating Engineering Society. Vol. 13, pp. 368-371 (1984).

(11) Kinameri, K., Akazawa K. and Awata, M. : "The Monte Carlo Method in the Predetermination of a Luminous Intensity Distribution", J. Light \& Vis. Env., Vol. 10, No. 2, pp. 57-66 (1986).

(12) Kajiyama, H. and Kodaira, S. : "An Illuminance Analysis in Partitioned Spaces Using the Monte Carlo Method", Journal of the Illuminating Engineering Society. Vol. 19, pp. 93-108 (1989).

(13) Aoki, Taniguchi and Irikura : "Computer Graphical Simulation of Light Signal System Including Light Scattering by Atmospheric Particles-Accuracy of Monte Carlo Simulation of Lamplight Scattering", J. Illum. Engng. Inst. Jpn., Vol. 80, No. 2, pp. 21-36 (1996).

(14) Uchida and Ohtani : "An Examination on the Illuminance Calculation within the Shadow by Task Ambient Lighting", J. Illum. Engng. Inst. Jpn., Vol. 81, No. 5, pp. 85-88 (1997).

(15) Lupton, M. J., Leung, A. S. M. and Carter, D. J. : "Advance in Lighting Design Method for Non-empty Interiors: A Review", Lighting Research and Technology, Vol. 28 No. 1, pp. 30-41 (1996). 
(16) Embrechts, J. J. : "Light Reflection model for lighting applications", Lighting Research and Technology, Vol. 27 No. 4, pp. 231-241 (1995).

(17) Born, M. and Wolf, E. : "Principles of Optics", Oxford Pergamon, second edn pp. 36-70 (1959)

(18) Jenkins, F. A. and White, H. E. : " Fundamentals of Optics", New York McGraw-Hill, third edn pp. 446534 (1965).

(19) Suzuki, M., Yoshimura, N., Kimura, O. and Awata, M. : "Monte Carlo Simulation for Color Changes Caused by an Inter-reflection Light", J. Illum. Engng. Inst. Jpn., Vol. 78, No. 2, pp. 65-71 (1994).

(20) Chen, L., Suzuki M. and Yoshimura, N. "Simulating Reflection Characteristic of Inspected Object in Automatic Inspection System by Monte Carlo Method", J. Light \& Vis. Env., Vol. 22, No. 1, pp. 4753 (1998).

(21) JIS Z8120, "Optics Terminology" (1986).

(22) Hopkins, H. H. : "The Frequency Response of a Defocused Optical System", Proc. Soc. Vol. 231, pp. 91-103 (1955).

(23) Fitzgerrell, A. R. Dowski, Jr. E. R. and Cathey W. T. : "Defocus Transfer Function for Circularly Symmetric Pupils", OSA. Applied Optics, Vol. 36, No. 23/10, pp. 5796-5804 (1997).

(24) Papoulis, A. : "Ambiguity Function in Fourier Optics", J. Opt. Soc. Am. Vol. 64, pp. 779-788 (1974)

(25) Bartelt, H., Ojeda-Castaneda, J. and Enrique, E. S. : "Misfocus Tolerance Seen by Simple Inspection of the Ambiguity Function", Appl. Opt. Vol. 23, pp. 26932696 (1984).

(26) Hanai, E., Suzuki M. and Yoshimura, N. : "A Study on the Optical Illumination of Image Equipment by Monte Carlo Method", J. Illum. Engng. Inst. Jpn., Vol. 79, No. 11, pp. 1-7 (1995).

(27) Uchida, A. and Ohtani, Y. : "Prediction of the shadow characteristics under the Task Ambient Lighting", Proc. LUXPACIFIC'97, pp. A7-A10, October 13-16 (1997) Nagoya, Japan. 